\title{
User perceptions of Online Public Library Catalogues
}

\author{
Elahe Kani-Zabihi ${ }^{*}$, Gheorghita Ghinea, Sherry Y. Chen \\ Department of Information Systems, Computing, Brunel University, Uxbridge, Middlesex UB8 3PH, UK
}

\begin{abstract}
Online Public Library Catalogues (OPACs) are widely used electronic library catalogues giving a wealth of remote access to library information resources. Users should be involved early in the OPAC development cycle process in order to ensure a usable and functional interface, as the integration of user-defined requirements of OPACs, along with the other Human-Computer Interaction considerations, offer a better understanding of user perceptions and expectations respect of OPACs, ultimately resulting in truly user-centred OPACs. Accordingly, the purpose of this study was to establish user suggestions for a typical OPAC application's functionality and features. To this end, an experiment was undertaken to find out the type of interaction features that users prefer to have in an OPAC. The study revealed that regardless of users' Information Technology (IT) backgrounds, their functionality expectations of OPACs are the same as users are expecting OPACs to facilitate easier ways to achieve their tasks. However, based on users' previous experiences with OPACs, their requirements with respect to specific features may change.
\end{abstract}

Keywords: OPAC, Electronic Library Catalogue; User study; User interfaces; User-centred design; Participatory design; Digital libraries

\section{Introduction}

Physical libraries in most developed countries are dependent on computer networks to provide access to local information resources. Hence, many institutions have used electronic library catalogues to allow remote access to the available resources. Today, electronic library catalogues over the Internet are known as OPACs. An OPAC is an online database which has an index of all library resources (in forms of text, audio and video), available in any institution. A good example of such institutions is given by universities, where students and academic staff usually use OPACs in order to search for resources [16]. As well as having the benefit of accessing a library's information remotely and saving time for a library's users, OPACs have also been shown to improve the communication between subject librarians and cataloguers $[17,6]$.

Although there has been considerable investment by academic and research libraries targeting online resources, with academic libraries in the United Kingdom spending around $£ 25$ million on electronic resources [9], there are, however, still issues outstanding in respect of the usability of these systems. For example, some OPAC systems are still not linked to other electronic resources, such as e-journals. Indeed, most OPACs do not have this facility for online users, and, once users have found the location of a book, the procedure of how to get hold of the book is vague [5]. There is, moreover, an issue which is most important of all, namely that young information seekers prefer to use other Internet resources such as search engines rather than their institutions' OPAC. This trend was confirmed by the results of a survey carried in 2001-2003 on further education (FE) colleges in UK, which showed that FE students do not use OPACs as much as they use other sources of information such as Internet 
search engines or organisational websites. Therefore researchers are eager to assess user satisfaction with library services and for this they have used both qualitative and quantitativeled studies to measure user levels of satisfaction with library services [24]. Moreover, as library managers are enthusiastic to know what differences their library services make to Learning, Teaching and Research (LTR), there have been an increasing number of studies on different approaches and methods for measuring the impact of these services on academic life $[19,7]$.

One of the premises of our research is that by involving end users in the OPAC design process, uncertainties to do with the efficacy of OPACS on LTR shall reduce, as designers of the library services are the user themselves. Accordingly in this paper we argue that, in designing and implementing OPAC user interfaces, users' abilities and experiences have to be taken into account. To this end, the study presented here investigates the functionality and features of OPACs from users' viewpoints with the aim of using these as future user requirements in developing OPACs. In particular, it focuses on how users' abilities and experiences influence their perceptions with respect to the interaction features provided by the OPAC. Accordingly, the structure of the paper is as follows: while section 2 presents related work, section 3 details the experimental study undertaken as part of our research. Section 4 then presents results, whilst conclusions and possibilities for future work are identified in section 5 .

\section{Background}

A close look at how users interact with OPACs helps to understand users' needs and consequently to design these systems in a way appropriate to satisfy its users. For instance, most users prefer to have less text on screen and more icons instead [5]. Moreover, users expect to see images of materials available on OPACs [8]. In updating and introducing a new version of OPAC services, users should be comfortable with the new version and should not struggle to do their task and be able to "find what they need, when they need it and in a form they want it" [6]. Designers of these systems should therefore be fully aware of users' skills and abilities.

Designers, however, are more likely to be successful in designing interfaces if they spend time with users, observing how they work. This process of interaction between designers and users is known as 'user and task analysis' [14] and has been so far a methodology insufficiently used in the design of OPACs. This in spite of its obvious advantages, for observing people in action can give the designer useful information on the usability of systems [22].

In fact usability has been shown to be of critical importance in achieving the satisfaction of users [11]. As for usability requirements of OPACs themselves, previous research [3] has confirmed that such systems should be useful and useable. In this context, the former term entails that information is made available to users in a suitable format at their requested time, while the latter captures requirements in respect of OPACs being: efficient and effective enough to satisfy the user; easy to learn; and able to support the user in his/her interactions with the system. Indeed, such considerations drive us to the conclusion that users' needs must be considered from the earliest stages and at the deepest levels of design of such systems.

There is a substantial body of research which targets users' interactions with OPAC interfaces. However, most existing user studies have mainly focused on OPACs' search 
engine's capabilities $[2,12]$ as well as on identifying user retrieval methods $[23,21]$, so that users are able to find the information they need with minimal effort. Whilst such research disregards the fact that environmental conditions affect people's behaviour more than their personal goals [4], an even more important omission, in our opinion, is that they implicitly assume that a good interface design has been executed in the first place. However, good interface design should ensure that OPACs' interfaces are friendly enough to deliver user satisfaction with the quality of their results first time round, irrespective of environmental conditions and any potential inhibiting influences these might exert.

It is thus expected that taking users' needs into full consideration shall increase the efficiency of OPACs and make them more usable and accepted by users. This can be done by actually involving users in the design stage and research shows that involving users in the development stage has resulted in delivering usable systems. Moreover, user involvement has been shown to result in user satisfaction at the end [18]. Although there are different approaches to this, in our study we are particularly interested in introducing elements of usercentred design and participatory design.

User-centred design methods focus on users through planning, design and development of a product, since only users can provide "sufficient knowledge and understanding of the context of use". In a survey of user-centred design practice, conducted by Vredenburg et al. [25], results showed that there was improvement in product usability and that user-centred design methods are extensively used in industry. Moreover participatory methods allow all participants (users, designers, and developers) to contribute equally and influence design. Therefore, users as well as designers and developers have an important impact in the product outcome [13].

In involving users in OPAC design, irrespective of whether one employs a user-centred design method or participatory method, it is however important to co-opt users with a variety of IT backgrounds, as their use of OPACs is very much dependent on their previous knowledge and experiences in IT. Support for this was shown in a study measuring online (database) searching experiences, conducted by Hsieh-Yee [15], which involved two user groups. The first was made up of 32 professional online searchers (who had at least one year of search experience or had taken courses on online searching), whilst the second comprised 30 novice searchers (who had little or no search experience and had not taken any courses on online searching). This particular study investigated how people use their search methods to search an online database and also whether their search experience and subject knowledge had an effect on their searching methods. The conclusion of this study showed that in fact both the subject expertise and search experience had an effect on the search method employed by users.

From a related but a different perspective, a study carried by Fields et al. [10] observed librarians while helping students who did not have sufficient skills to find the information they need using the catalogue. Two librarians were videotaped and were asked to think out loud whilst they were interacting with the students. Here again, the conclusion of the study was that expert users are more successful at finding information than non-experts, thus supporting our view that OPAC design has to take into account user IT skills.

Moreover, the report produced by Tenopir et al. [23], which gives an overview of some 200 research studies on the use and users of electronic library resources, has also highlighted different user behaviours with electronic resources. The study concluded that high school and 
undergraduate students will often use library electronic resources if they have been asked to use it for a specific assignment. It has also highlighted that despite their differences in the type of education they had and their needs, both high school and undergraduate students preferred to access electronic resources through the library from home. However, graduate students, and particularly PhD students, are the main users of these services.

As opposed to previous studies [16, 20, 1, 21] in the area, which have mainly focused on information retrieval and usability issues in OPACs, the purpose of the research reported in this paper is to determine end user opinions and suggestions in respect of existing OPAC features and functionalities; and also to explore how these are influenced by the particular IT background of a user. In so doing, we hope to take a first step towards integrating user expectations and preferences in OPAC design.

\section{Experiment}

As mentioned earlier, just like in the case of any other software system, understanding users and their needs is important in the early stage of the OPAC design process. We have therefore undertaken an experiment to understand users' expectations and preferences of OPACs.

In keeping with previous research in the area [6] which has highlighted the use of observations, interviews, experiments and transaction log analyses of end users, our study was made up of two parts. In the first, after completing a user IT profiling questionnaire, users were observed while undertaking four tasks using these different OPACs. In the second, users were asked their opinions in respect of OPAC features and functionalities via a semistructured questionnaire.

\subsection{Participants}

We were particularly interested in how the level of users IT sophistication impacted the quality of suggestions for OPACs. Accordingly, we polled users having a variety of IT knowledge backgrounds, with a total of 54 users ( 31 female and 23 male), aged between 19 and 49 years old, from diverse academic backgrounds (Fig. 1) participating in our study.

Users were grouped into three different categories (novice, intermediate and advanced), with an equal number in each category. In order to determine in which particular category a user belonged, each participant in our study had to complete a questionnaire (Table 1). Participants, who answered "yes" to the first three questions and "no" to the rest were categorised as being in the novice category; if participants answered "yes" to any of the three further questions, they were deemed to be in the intermediate category. Further answering "yes" to either question 9 or 10, in addition to the first eight questions, made a user part of the advanced category.

\subsection{Procedure}

Since OPACs are widely used in academic institutions, and particularly in universities, our study involved users interacting with three OPACs from London universities: Brunel University; City University; King's College London University (KCL). These OPACs were chosen as they had differentiating or distinctive functionality features which were beneficial for our experiment. 


\subsubsection{OPACs}

The Brunel OPAC (Fig. 2) updated its features in 2004-05, with users being now able to see more details about an item in the OPAC, such as the abstract of a book. However, in introducing new interfaces, tasks such as placing a hold on an item have arguably became more complex. To examine this, task one of our study targets exactly this facility of the Brunel OPAC. As anecdotal evidence pointed to the possibility that the Brunel OPAC help service feature is poor, in the second task of our study we asked users to find an inter-library loan service (not provided by the OPAC), and thus explore whether the support available is sufficient to guide users in this way.

As opposed to Brunel's, the City OPAC (Fig. 6) allows users to search for books using the ISBN number from the OPAC home page, via a drop down menu from the search bar. Moreover the OPAC displays all books nearby the shelf of the searched item. Users are therefore able to see other books which might also be helpful to them, much in the same way as they would pick a book from its shelf in a physical library. Task three was thus allocated to City to find out whether users are able to use these services.

Finally, task four concerned the KCL OPAC (Fig. 10). Services provided by this OPAC were relatively different to those used in Brunel and City, with users having access to a rich functionality array. For instance, here users were able to save their searches; view their previous searches; add an item to 'Basket' for future use; email the content of an item to their email account; see the details of recently searched item; search for items using different category such as DVD, Books, music and etc; and finally users were able to clear 'Basket' and 'Previous Searches' lists by clicking on 'End session' button on the screen. Task four, as shall be shown below, explored whether users would use the whole functionality spectrum at their disposal.

\subsubsection{Tasks}

The tasks that users had to undertake in our study were:

Task one: Search the library for any book, which contains material on Distributed Systems and Java. Once you have located such a book, place a 'hold' on it.

Task two: Find out whether you can order an article through the inter-library loan service. Task three: Search for the book having ISBN number 0471346098. When you find it, look for at least four other books within the same subject area (the Java programming language) located on the same physical shelf as this book.

Task four:

Step 1 - Search for the book entitled XML in a nutshell; view full details of the book; add the book to the 'Basket' so you can view it again; email the details of the book to yourself.

Step 2 - Search for The Godfather DVD; find out who starred in the movie; add it to the 'Basket'.

Step 3 - Check the list of items you have added to 'Basket'.

Step 4 - See the list of searchers you have done so far.

Step 5 - Go to the result list of the search for the film The Godfather.

Step 6 - Clear all your searches to start a new search with one click. 
All users were observed by the same single person, who recorded their action and their achievement. The observer also made sure each user understood the terminology used in the questionnaire and the purpose of each task given to them. Users were also asked to think out loud while interacting with OPACs.

To find out users' opinions about each task using the related OPAC, we asked them to complete a questionnaire (Table 2) after accomplishing each task. This contained a set of five statements on which the user expressed their opinion on a five-point scale (strongly agree, agree, natural, disagree, and strongly disagree). Each statement described whether the user was happy with the way the task is delivered by the OPAC or if the user would have preferred to do the task using a physical library instead.

Having experienced three different types of OPACs, the last part of the study involved users completing a questionnaire soliciting their views on OPACs. Accordingly, we administered a questionnaire soliciting both quantitative and qualitative data, via the use of both closed and open-ended questions. The questionnaire itself thus contained two parts, the first of which solicited user opinions on features that they would prefer the OPACs to contain (Table 3). Finally, in order to find out users' specific preferences in terms of features and user interfaces of OPACs, the last part of the experiment involved users replying to an open-ended questionnaire soliciting their views on OPACs in general (Table 7).

\subsubsection{Data Analysis}

Data collected was analysed with the Statistical Package for Social Sciences (SPSS), version 11.5. The t-test and Analysis of Variance (ANOVA) were applied to identify potential significant differences between the three user types. The significance value used for this study was $\mathrm{p}<0.05$.

\section{Results}

\subsection{Users' interaction with OPACs}

As mentioned above, participants were asked to use three OPACs while their interactions were observed. From the outset, it must be remarked that almost $76 \%$ of users $(n=41)$ did not accomplish all tasks correctly - and this does not exclude experienced users. Moreover, the observer noted that users often blamed their own lack of knowledge of IT when they did not achieve a task, leading further support to including user IT profiles in the design of OPACs. We now proceed to describe user interaction with the three OPACs in detail.

\subsubsection{Brunel OPAC}

The Brunel OPAC updated its interfaces in the 2004-2005 academic year. The new version has more functions available and displays more details about a selected book than the earlier version used to (Fig. 2). However, participants who had used the catalogue in previous years commented that they preferred the old version, as it was less complicated, and tasks such as placing a hold on a book had previously been much more straightforward. 
An ANOVA on our study data shows that all three different types of users said they will use the Brunel OPAC again ( $\mathrm{p}=0.012, \mathrm{~F}=4.801)$, even though $31.48 \%$ of users $(\mathrm{n}=17)$, mostly novice, could not accomplish Task 1 (Fig. 5). Fig. 3 shows the average credit given by users in their responses to the questionnaire (Table 2) and Fig. 4 shows the responses from each category.

As can be seen apart from Task1-Q3, there was general agreement ( $\mathrm{df}=53, \mathrm{p}=0.000)$ among all users as regards their ranking opinion of each task. Although all three types of users indicated it was easy to do this task, they did prefer to use a physical library for this task, especially advanced and intermediate users. This may point towards the fact that the OPAC did not satisfy users' needs and their expectations were not fulfilled.

Surprisingly, all users surveyed in our study indicated that they found it easy to familiarise with the OPAC (Task1-Q6). Hence, although users were confident using the OPAC, they were not happy with the way Task 1 could be accomplished in the OPAC, as the link to placing a hold on the book was not easy to find. This is especially the case with intermediate users, who were not in favour of using the OPAC to place a hold on a book.

$72.22 \%$ of novice users $(n=13)$ struggled to find the 'Place Hold' link (Fig. 5). These users suggested that it could have been better if this option was available at the result page and this link should be placed in a more obvious place on the page. A novice user commented that:

\section{"The system does not help nor make it easy to find this link".}

The observer also noted that $18.52 \%(\mathrm{n}=10)$ of users gave up using the Help function after their first attempt. Indeed the rest did not notice the link (Fig. 5) and users commented that the Help function did not guide them and should be more supportive.

In addition to novice users, the other two types of users also commented that functions such as 'Place Hold' that are frequently used in OPACs should be visible and strategically located for users to see. Moreover it would have been better if this option followed the main information (book's details). They also commented that this option was not clearly defined, and that these interactions should be in the form of a button. Table 4 gives overall comments given by all users.

\subsubsection{City OPAC}

As opposed to the Brunel OPAC, users were much more comfortable interacting with the City OPAC (Fig. 6). Fig. 7 shows the average scores given by users in their responses to the questionnaire (Table 2).

Apart from Q3 and Q4 (Table 2), there was general agreement $(\mathrm{df}=53, \mathrm{p}=0.000)$ among all users as regards their ranking opinion of each task. It seems that users did not all agree whether the help provided was sufficient and if they would prefer to use the OPAC in future. However, all users agreed that it was easy to use the OPAC and to read the information provided. Our results show that Intermediate users seemed to like this OPAC more than users from the other two categories (Fig. 8). 
An option to search for books by ISBN number was available under a drop down menu list on the first page of searching. Only six users (two from each category) could not find this option and hence did not find the book at the first attempt (Fig. 9).

$88.88 \%(\mathrm{n}=48)$ of users managed to find the book on the same shelf number, by clicking on the right link (Fig. 9). The rest, however, either tried searching by the shelf number or subject name. A novice user said:

"It was not immediately obvious that clicking on the item would bring up nearby items on the shelf".

An advanced user commented:

"The ability to browse items on a shelf is very useful since this replicated what we are able to do in the physical library. Some description of what the link did to enable us to browse in this way would be useful, as this is not particularly clear".

Moreover, as Table 5 highlights, overall comments given from users across all categories, were mainly positive.

\subsubsection{KCL OPAC}

The result from the KCL OPAC (Fig. 10) shows that users were also happy with the interfaces of this catalogue. Indeed one user commented that:

"It was easier to follow than the other two OPACs".

Fig. 11 shows the average credit given by users in their responses to the questionnaire (Table 2). Here, we have a similar results profile to those obtained in Task 1 and Task 3 . Thus, apart from Q3 (Table 2) there was general agreement ( $\mathrm{df}=53, \mathrm{p}=0.000)$ among all users as regards their ranking opinion of each task. More advanced users were in favour of using the KCL OPAC than the previous ones.

However, it must be said that although all users from three categories have said tasks done on this OPAC were easy (Fig. 12), and it was easy to be familiar with the OPAC, not all users accomplished their tasks correctly. Thus $35.19 \%$ of users $(n=19)$, mainly from the novice category, could not find the 'Add to basket' link (Fig. 13). These users were clicking on 'Basket' button instead, which was at the top of the page. One typical comment given on this function was that the terminology 'Basket' or 'Add to basket' is used for purchasing items on e-shopping websites rather than OPACs. Therefore, different phrases should be used for these functions.

$90.74 \%$ of users $(n=49)$ did not find the DVD search option under 'Subset' menu. These users searched for the DVD in much the same way as they searched for a book. It was not obvious to users that the 'Subset' button contains the list of different materials available in the OPAC e.g. DVD, Books, Journals. Some users looked for a DVD search option under the drop down menu on the first page. Only four advanced users and one intermediate user managed to find this option (Fig. 13). 
Fig. 13 shows that the majority of users managed to view the content of the 'Basket'. Users who did not find the option, were from all three categories. Thus, one advanced user commented:

"I cannot see the reason for having a 'Basket'. Items are stored by their titles and other details, so this is an unnecessary function. Also the terminology basket is more linked to purchasing items than storing them for later retrieval".

Another advanced user said:

“... adding items to 'Basket' was unnecessarily complicated by having to add a description (of what we have to add)".

However, $35.19 \%$ of users $(n=19)$ did not click on the 'Previous Search' button which, was in the same style as the 'Basket' button (Fig. 13). This may indicate that users were not familiar with the phrase used and therefore could not make a connection between the labelled button and the task. One advanced user said:

"The 'Previous Search' screen was too cluttered and kept track of unnecessary combinations of searches".

A similar observation applies to the 'Results List' button (Fig. 13).

Another difficult task for users was when they were required to clear their previous searches using one click. Only $29.63 \%(n=16)$ of overall users employed the 'End Session' button, which was designed for this purpose (Fig. 13). An intermediate user said:

"The name given to this button does not define its functionality as I thought by clicking on it I might exit from the catalogue”.

In concluding, we remark that users were generally more confident in using the KCL OPAC than the other two and overall comments are shown in Table 6. Nonetheless, users commented that the KCL OPAC had introduced new features, some of which were unnecessary and rather confusing. Therefore the number of people satisfactorily achieving Task 4 was low.

\subsection{Users' preferences for OPACs}

Not all users preferred the KCL OPAC. Among all three types of users, a few said that they preferred the City OPAC as it was simpler to use. Another user commented that the catalogue had too many options and functions which required familiarity to use them all. In order to find out which OPAC had the best impact on users, they were asked to identify their favourite one and specify their reasons. The results showed that $9.25 \%$ of users $(n=5)$ preferred to use the Brunel OPAC as they had used it before and were therefore more familiar with it. $27.78 \%$ of users $(n=15)$ preferred the City OPAC due to such reasons as: there being less information on screen for easier searching; it was straightforward and simple to use; it was comfortable to navigate through the site; the task was done without any problems; search options were labelled clearly; the form was simple; the page looked familiar. However, the highest 
percentage of users preferred the KCL OPAC $(37.04 \%, n=20)$. Users said that this catalogue is friendlier than the other two; the use of buttons made it easy to use; it has unambiguous option names; there is more information available on a book. Nonetheless, $27.78 \%$ of users $(n=15)$ had no preference as they considered some functions in each of the applications useful while finding some interfaces that were not user friendly. Therefore they felt their primary requirements were not fully satisfied by any of them.

Users were asked to write down the five most important requirements that they felt OPACs should satisfy. The majority of users were concerned about the easiness of using a OPAC. Hence, according to them, being able to use a OPAC easily without too much effort is an important requirement that should be fulfilled. The other two important requirements were that OPACs should be able to offer the same basic services as physical libraries, such as renewing books, borrowing books, searching for books, and that they should have a reliable search engine capable to provide updated information. Furthermore, users commented that OPACs should be user friendly, provide reliable information and sufficient Help services. Less important requirements mentioned by some users concerned issues such as the appearance of OPACs and having a personal profile.

The second part of the questionnaire that users had to complete, following their interactions with the three OPACs used, consisted of suggestions as to the features and functionality that users themselves would like to see in OPACs. We have grouped users' responses into three sections: functionality, interface/usability, and content (Table 7).

Our results show that as far as functionality requirements are concerned, users indicated that they would like to see all services available in a physical library also available in an OPAC. Moreover, users would like to be able to search the content of all books. User responses regarding interfaces of OPACs mostly concerned the way they could access links. Accordingly, users indicated that they prefer to use more icons or buttons rather than simple links. It thus came as no surprise that users indicated their preference for visual-based OPAC interfaces, rather than ones based predominantly on text. Lastly, as far as OPAC content is concerned, expressed user opinion was that OPACs should contain a variety of media (pictures, music clips and videos).

\section{Discussion}

Comments given by users in this experiment once again have proved the importance of employing fundamental usability concepts in good systems design. Surprisingly, users from both the novice and intermediate categories - who are unaware of these concepts - have clearly highlighted the need of using them in OPACs. Worryingly, the OPACs used in our study seem to have lapses in respect of these elementary usability properties.

Another key observation has to do with the importance of the OPAC searching facility to users, to the detriment even of the layout and interfaces, for users surveyed in our study suggested that OPACs be easy to learn and reliable in terms of obtaining search results $-\mathrm{a}$ feature which OPAC designers must consider in the design of such systems.

We also observed that only a fraction of the users who participated in our study managed to complete all tasks, and those that did were the more experienced users. This may indicate a lack of understanding of OPAC users and their needs - indeed, if OPACs are designed for use, they should be so such that all types of users are taken into account. 
Users demanded to see more flexible interfaces. Furthermore, they emphasised that important features should be clearly presented on screen so they can be easily seen, without having to be searched or browsed for. For example in Task 4: Step 5, the DVD submenu where users could access to all DVDs available, was not used by the majority of users (the submenu was invisible to users; hence its functionality was limited). Users also seemed to prefer less information on their screen. User comments and suggestions expressed in our study convey elementary HCI ideas and requirements; what is striking, though, is that these have been found lacking in all the three OPACs used in our study.

Moreover, although the majority of participants in our study agreed that using these OPACs was easy, in practice these users had difficulties using the full set of functionality features of the three OPACs. This adds further support to our belief that end users from a variety of IT backgrounds should be involved early in OPAC design, not only in respect of requirements definition, but also be observed in their interactions with OPAC prototypes, so that functionality is streamlined and the user experience improved.

\section{Conclusions}

In this paper we have reported the results of a study which sought to explore the gap between users' self-reported OPAC requirements and the functionality and usability of such systems in practice.

We believe that the integration of user-defined requirements of OPACs, along with other Human-Computer Interaction considerations, offers a better understanding of user perceptions and expectations with respect to OPACs and ultimately result in the design of truly usercentred OPACs. Accordingly, in our work, as opposed to previous studies in the area, which have mainly focused on information retrieval and usability issues in OPACs, we have concentrated on eliciting users' opinions regarding features that OPACs should have from users with a wider spectrum of IT skills, ranging from novice to advanced users.

This study was a first step towards the design of an OPAC based on what users want. It has clearly highlighted the need to improve the current status and the user experience of information access. For this, it is essential that user requirements form an integral part in the design and development part user-centred OPACs, and this forms the focus of our future work.

\section{Acknowledgements}

We are grateful to all 54 participants who took part in this study.

\section{Appendix}




\section{References}

[1] Agosti, M. and Masotti, M. (1992), "Design of an OPAC database to permit different subject searching accesses in a multi-disciplines universities library catalogue database". N. Belkin, P. Ingwersen and A.M. Pejtersen (Eds), Proceedings of the 15th annual international ACM SIGIR conference on Research and development in information retrieval, June $21-24$, 1992, pp. 245-255.

[2] Babu, R.B. and Tamizhchelvan, M. (2003), "An investigation into the features of OPACs in Tamil Nadu (India)”. Library Review, 52(6), pp. 257-267.

[3] Blandford, A. and Buchanan, G. (2003), "Usability of digital libraries: a source of creative tensions with technical developments", IEEE-CS TC Bulletin, available at: http://www.ieeetcdl.org/Bulletin/summer2003/blandford/blandford.html.

[4] Blandford, A. and Stelmaszewska, H. (2001), "Shooting the Information Rapids", Vanderdonckt, Blandford and Derycke, (Eds), IHM-HCI2001, pp51-54.

[5] Bordeianu, S., Carter, C.E. and Dennis, N.K. (2000), "Delivering electronic resources with Web OPACs and other Web-based tools: needs of reference librarians", Reference Service Review, 28(2), pp. 111-118.

[6] Borgman, C.L. (2003), "The invisible library: paradox of the global information infrastructure". Library Trends, 51(4), pp. 652-674.

[7] Brophy, P. (2002), "The evaluation of public library online services: measuring impact". People's Network Workshop Series. Issue Papers No.1.

[8] Brown, C. (2006), "Digitisation projects at the University of Dundee Archive Services", Program: electronic library and information systems, 40(2), pp. 168-177.

[9] Creaser, C., Hamblin, Y. and Davies, J.E. (2006), "An assessment of potential efficiency gains through online content use", Program: electronic library and information systems, 40(2), pp. 178-189.

[10] Fields, B., Keith, S. and Blandford, A. (2004), "Designing for Expert Information Finding Strategies", S. Fincher and P. Markopoulos (Eds), People and Computers XVIII Design for Life, Springer pp89-102.

[11] Flavián, C., Guinalíu, M. and Gurrea, R. (2006), “The role played by perceived usability, satisfaction and consumer trust on website loyalty". Information and Management, 43(1), pp. $1-14$.

[12] Fox, E.A., France, R.K., Sahle, E., Daoud, A. and Cline, B.E. (1993), "Development of a modern OPAC From REVTOLC to MARIAN", R. Korfhage, E. Rasmussen and P. Willett (Eds), June 27 - July 01 1993, ACM Press pp248-259.

[13] Gulliksen, J., Boivie, I. and Göransson, B. (2006), "Usability professionals-current practices and future development”, Interacting with Computers, 18(4), pp. 568-600. 
[14] Hackos, J.T. and Redish, J.C. (1998), “User and Task Analysis for Interface Design”, 1 edn. New York: Wiely.

[15] Hsieh-Yee, I. (1993), "Effects of Search Experience and Subject Knowledge on the Search Tactics of Novice and Experienced Searchers", Journal of the American Society for Information Science, 3(44), pp. 161-174.

[16] Kan, M. and Poo, Danny C. C. (2005), "Detecting and supporting known item queries in online public access catalogs", Proceedings of the 5th ACM/IEEE-CS joint conference on Digital libraries, June 7-11 2005, ACM Press (New York, USA) pp91-99.

[17] Kennedy, P. (2004), "Dynamic web pages and the library catalogue", The Electronic Library, 22(6), pp. 480-486.

[18] Kujala, S. (2003), "User involvement: a review of the benefits and challenges", Behaviour \& Information Technology, 22(1), pp. 1-16.

[19] Markless, S. and Streatfield, D. (2006), "Gathering and applying evidence of the impact of UK university libraries on student learning and research: A facilitated action research approach”. International Journal of Information Management, 26(1), pp. 3-15.

[20] McMullen, S. (2001), "Usability testing in a library Web site redesign project", Reference Service Review, 29(1), pp. 7-22.

[21] Nordlie, R. (1999), "User revealment — a comparison of initial queries and ensuing question development in online searching and in human reference interactions". Proceedings of the 22nd annual international ACM SIGIR conference on Research and development in information retrieval, pp. 11-18.

[22] Stelmaszewska, H., Blandford, A. and Bryan-Kinns, N. (2001), "Use of multiple digital libraries: a case study", Fox, E.A. and Borgman, C.L. (Eds), JCDL 2001, June 24, 2001, ACM pp179-188.

[23] Tenopir, C., Hitchcock, B. and Pillow, S.A. (2003), "Use and Users of Electronic Library Resources: An Overview and Analysis of Recent Research Studies", Council on Library and Information Resources.

[24] Urquhart, C., Thomas, R., Spink, S., Fenton, R., Yeoman, A., Lonsdale, R., Armstrong, C., Banwell, L., Ray, K. and Coulson, G. (2005), "Student use of electronic information services in further education”. International Journal of Information Management, 25(4), pp. 347-362.

[25] Vredenburg, K., Mao, J., Smith, P.W. and Carey, T. (2002), “A survey of User Centred Design in practice", pp471-478.

\section{Fig. Captions}

Fig. 1 - Participants in the experiment

Fig. 2 - Brunel University - OPAC

Fig. 3 - Users' responses to Task 1

Fig. 4 - Responses to Task 1 by each category

Fig. 5 - Number of people who used Help option and placed Hold on the book 
Fig. 6 - City OPAC

Fig. 7 - Users' responses to Task 3

Fig. 8 -Responses to Task 3 by each category

Fig. 10 - KCL OPAC

Fig. 11 - Users' responses to Task 4

Fig. 12 - Responses to Task 4 by each category

Fig. 13 - Number of users who used KCL OPAC features

\section{$\underline{\text { Table Captions }}$}

Table 1 - User profile questionnaire

Table 2 - OPACs' Questionnaire

Table 3 - OPACs' features

Table 4 - Users' comments on Task 1

Table 5 - Users' comments on Task 3

Table 6 - Users' comments on Task 4

Table 7 - Users' suggestions for future OPACs systems 